\title{
Clinical guidelines for burosumab in the treatment of XLH in children and adolescents: British paediatric and adolescent bone group recommendations
}

\author{
Raja Padidela', Moira S Cheung ${ }^{2}$, Vrinda Saraff ${ }^{3}$ and Poonam Dharmaraj ${ }^{4}$ \\ ${ }^{1}$ Royal Manchester Children's Hospital and Faculty of Biology, Medicine and Health, University of Manchester, Manchester, UK \\ 2Evelina London Children's Hospital, London, UK \\ ${ }^{3}$ Birmingham Women's and Children's Hospital, Birmingham, UK \\ ${ }^{4}$ Alder Hey Children's NHS Foundation Trust, Liverpool, UK
}

Correspondence should be addressed to R Padidela: raja.padidela@mft.nhs.uk

\begin{abstract}
X-linked hypophosphataemia (XLH) is caused by a pathogenic variant in the PHEX gene, which leads to elevated circulating FGF23. High FGF23 causes hypophosphataemia, reduced active vitamin $\mathrm{D}$ concentration and clinically manifests as rickets in children and osteomalacia in children and adults. Conventional therapy for XLH includes oral phosphate and active vitamin $D$ analogues but does not specifically treat the underlying pathophysiology of elevated FGF23-induced hypophosphataemia. In addition, adherence to conventional therapy is limited by frequent daily dosing and side effects such as gastrointestinal symptoms, secondary hyperparathyroidism and nephrocalcinosis. Burosumab, a recombinant human IgG1 MAB that binds to and inhibits the activity of FGF23, is administered subcutaneously every 2 weeks. In clinical trials (phase 2 and 3) burosumab was shown to improve phosphate homeostasis that consequently resolves the skeletal/non-skeletal manifestations of XLH. Burosumab was licensed in Europe (February 2018) with the National Institute for Health and Care Excellence, UK approving use within its marketing authorisation in October 2018. In this publication, the British Paediatric and Adolescent Bone Group (BPABG) reviewed current evidence and provide expert recommendations for care pathway and management of XLH with burosumab.
\end{abstract}

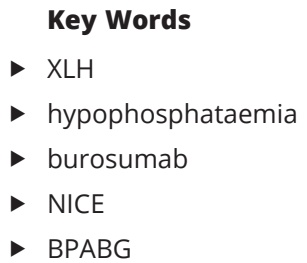

Endocrine Connections (2020) 9, 1051-1056

\section{Introduction}

$\mathrm{X}$-linked hypophosphataemia (XLH) is a rare genetic condition affecting phosphate homeostasis, which results in debilitating bone deformation from an early age with lifelong consequences. The condition is caused by a pathogenic variant in the PHEX gene, as a result of which serum FGF23 levels are elevated, resulting in hypophosphataemia.

Low serum phosphate reduces hydroxyapatite formation causing osteomalacia and leads to the failure of apoptosis of hypertrophied chondrocytes and mineralisation of growth plates causing rickets. These conditions lead to soft and often deformed bones, with skeletal abnormalities and short stature giving rise to lifelong pain, impaired physical function, and disability. Additionally, failure of mineralisation of dentine causes dental abscess, while the combination of bone deformities and reduced muscle function causes fatigue and limitations in mobility (1).

Conventional therapy for XLH has been implemented via supplementation with multiple daily doses of oral
This work is licensed under a Creative Commons Attribution-NonCommercial-NoDerivatives 4.0 elnternationab sicense ifica.com at 04/26/2023 07:32:03AM 
phosphate and active vitamin D analogues $(2,3)$ but does not specifically treat the underlying pathophysiology of elevated FGF23-induced hypophosphataemia. Oral phosphate produces only a transient increase in serum phosphate levels and the frequent daily dosing and gastrointestinal symptoms may compromise treatment persistence/compliance (2). In addition, increase in serum phosphate leads to reduction of serum ionised calcium causing secondary hyperparathyroidism and, if left untreated, tertiary hyperparathyroidism. Active vitamin D analogues can prevent hyperparathyroidism but increase the risk of nephrocalcinosis.

Burosumab is a recombinant human IgG1 MAB that binds to and inhibits the activity of fibroblast growth factor 23 (FGF23). By directly inhibiting excess FGF23, burosumab increases tubular reabsorption of phosphate from the kidney and, through the production of 1,25-dihydroxyvitamin D $\left(1,25(\mathrm{OH})_{2} \mathrm{D}\right)$, enhances intestinal absorption of calcium and phosphate (4). Trials, in 1- to 12-year-old children, have demonstrated that burosumab improves bone mineral metabolism, heals rickets, which leads to increased growth, mobility, and physical functioning and has the potential to significantly alter the natural history of the disease (5, 6). Its fortnightly subcutaneous dosing schedule eliminates the need for multiple daily doses of conventional therapy, and the drug is well-tolerated, avoiding the complications associated with conventional phosphate and vitamin D therapy (7).

The European Medicines Agency approved burosumab (brand name Crysvita ${ }^{\mathrm{TM}}$ ) on 19 February 2018 (https:// www.ema.europa.eu/en/documents/overview/crysvitaepar-summary-public_en.pdf) for the treatment of $\mathrm{X}$-linked hypophosphataemia with radiographic evidence of bone disease in children 1 year of age and older and adolescents with growing skeletons. It is administered as a subcutaneous injection every 2 weeks (https://www.ema. europa.eu/en/documents/product-information/crysvitaepar-product-information_en.pdf).

Burosumab has been reviewed by the National Institute for Health and Care Excellence (NICE) under their Highly Specialised Technology (HST) appraisal programme. An unrestricted recommendation was made on 10 October 2018, within the terms of its product licence, for conventional therapy naïve and experienced patients with XLH (https://www.nice.org.uk/guidance/HST8). This assessment was based on the latest clinical evidence including the UX023-CL301 study, a Phase III study evaluating the safety and efficacy of burosumab compared to conventional therapy in 61 paediatric patients aged 1 to $\leq 12$ years with XLH who had confirmed evidence of rickets. These results have now been published showing that treatment with burosumab improved renal tubular phosphate reabsorption, serum phosphorus levels, linear growth, physical function, and reduced pain and the severity of rickets in this group of children (5). A recent publication also showed that burosumab has a favourable safety profile, increases serum phosphorus, and improved rickets and prevented early declines in growth in children aged 1-4 years with X-linked hypophosphataemia (6).

There are few clinical guidelines on XLH providing recommendations specific to healthcare practice in the UK. Guidelines on the diagnosis and management of XLH have been produced by a group of clinical experts in the USA $(2,7)$. These guidelines provide specific recommendations for management of XLH in children and adults. This aligns with the proposals of an expert panel of the Japanese Society for Bone and Mineral Research (8) and guidelines from France (3). Crucially, all of these guidelines predate the availability of burosumab. There has however been a comprehensive recent international publication on clinical guidelines for XLH and this should be referred to for detailed aspects of XLH care and management in children and adults (1). Although the NICE recommendation for the use of burosumab has been published (https:// pathways.nice.org.uk/pathways/endocrine-nutritionaland-metabolic-conditions\#path=view $\% 3 \mathrm{~A} /$ pathways $/$ endocrine-nutritional-and-metabolic-conditions/ metabolic-conditions.xml\&content=view-node\%3Anodes$\mathrm{x}$-linked-hypophosphataemia) there is currently no peer reviewed clinical guidance on the management and best practice use of burosumab in children with XLH.

Following approval and the availability of burosumab for management of children and adolescents with XLH, the British Paediatric and Adolescent Bone Group (BPABG), a comprehensive group of clinicians managing children with bone disorders in the UK, have taken an opportunity to provide recommendations for the management of XLH to optimise clinical practice.

\section{Methods}

The recommendations set out in this paper were initially developed by the BPABG via an advisory board meeting for management of XLH using burosumab following EMA licensing and NICE authorisation for use within the NHS. The advisory board reviewed data from phase 2 and 3 clinical trials and took into consideration the schedule of events from research protocol. The authors formed a core group to finalise the recommendations.

This work is licensed under a Creative Commons Attribution-NonCommercial-NoDerivatives 4.0 International License ifica com at $04 / 26 / 2023 \quad 07: 32: 03 A M$ 


\section{Results and discussion}

We propose the following recommendations for the clinical management of XLH with burosumab in children and adolescents. As diagnosis and conventional therapy have already been addressed at length in the previously noted reviews, these recommendations will focus on updating these existing clinical practice recommendations in light of the licensing and NICE recommendation of burosumab.

\section{Initiation/cessation}

In Europe, burosumab is indicated and recommended for the treatment of XLH in patients with radiographic evidence of bone disease in children 1 year of age and older and adolescents with growing skeletons. In the USA, Food and Drug administration has approved burosumab for treatment of XLH in paediatric patients 6 months of age and older and adult. The NICE recommendation in UK is not population restricted beyond the indication of the EMA licence, so both newly diagnosed XLH patients and those currently on conventional therapy, irrespective of the disease severity, are eligible for treatment with burosumab. However, local guidance and reimbursement procedures will need to be taken into consideration to assess for eligibility.

Initiation of treatment for patients should occur once diagnosis is confirmed (through biochemical, radiographic evidence and genetic testing in the patient or a family member), provided that the patient is at least 1 year old but still within the age where significant bone growth is occurring. The decision to begin burosumab treatment in the UK should be agreed by the guardian/ patient, responsible clinician, and tertiary metabolic bone specialist. NHS England stipulates that burosumab should be initiated by a hospital Trust who is a member of the European Reference Network for Rare Bone Disease (ERN BOND). A list of centres is provided in Supplementary data (see section on supplementary materials given at the end of this article). To enable NHS England to monitor and reimburse the appropriate use of burosumab in line with NICE guidance, a formal Prior Approval Scheme called Blueteq (http://www.blueteq.com/) is required to be completed to confirm patient eligibility before a prescription for burosumab is initiated.

The decision to stop treatment should rest with the metabolic bone centre and should be based on evidence of slowing of growth towards final height. Since European license is limited to adolescents with growing skeleton, it is recommended that once skeletal growth velocity falls below $<2 \mathrm{~cm} /$ year, the decision to stop treatment should be considered and discussed, with a view to cessation of therapy. Radiographic evidence may be useful in informing this process.

\section{Initiating treatment}

Burosumab treatment should not be initiated unless serum phosphate concentration (measured after at least 4 $\mathrm{h}$ fasting) is below the reference range for age. Burosumab should not be given along with conventional treatment. Patients on conventional therapy should stop oral phosphate and active vitamin $\mathrm{D}$ analogues 7 days prior to commencing burosumab treatment (https://www.ema. europa.eu/en/documents/product-information/crysvitaepar-product-information_en.pdf).

Immediately prior to treatment initiation, fasting blood tests should be conducted to assess levels of serum phosphate, parathyroid hormone (PTH) and 25-hydroxyvitamin D $(25(\mathrm{OH}) \mathrm{D})$. A paired urine sample should be taken for urinary creatinine, phosphate and calcium from which tubular maximum reabsorption of phosphate (TmP/GFR) can be calculated. Any other appropriate radiological investigations and biochemical tests should be undertaken as necessary. Reference ranges are assay and laboratory specific and each laboratory should use age specific reference ranges for all biomarkers.

\section{Dosing}

Summary of Product Characteristics (SmPC) (https://www. ema.europa.eu/en/documents/product-information/ crysvita-epar-product-information_en.pdf) recommend burosumab treatment should begin at a starting dose of $0.8 \mathrm{mg} / \mathrm{kg}$ subcutaneously every 2 weeks, however, some patients are able to normalise serum phosphate and ALP concentrations on starting dose of $0.4 \mathrm{mg} / \mathrm{kg}$ and therefore it is our recommendation to initiate treatment on this dose. After commencing treatment, fasting serum phosphate levels should be monitored every 2 weeks during the first month, every 4 weeks for the following 2 months and thereafter as appropriate; fasting serum phosphate should be measured pre-dose on every occasion. We also recommend measuring fasting serum phosphate 4 weeks after each dose adjustment (Fig. 1). Peak serum phosphate level is achieved 7-11 days after injection (https://www. ema.europa.eu/en/medicines/human/EPAR/crysvita).

Dose titration should be performed with the aim of bringing fasting serum phosphate levels within the lower

This work is licensed under a Creative Commons Attribution-NonCommercial-NoDerivatives 4.0 Internationab License ifica com at $04 / 26 / 2023 \quad 07: 32: 03 \mathrm{Am}$ 


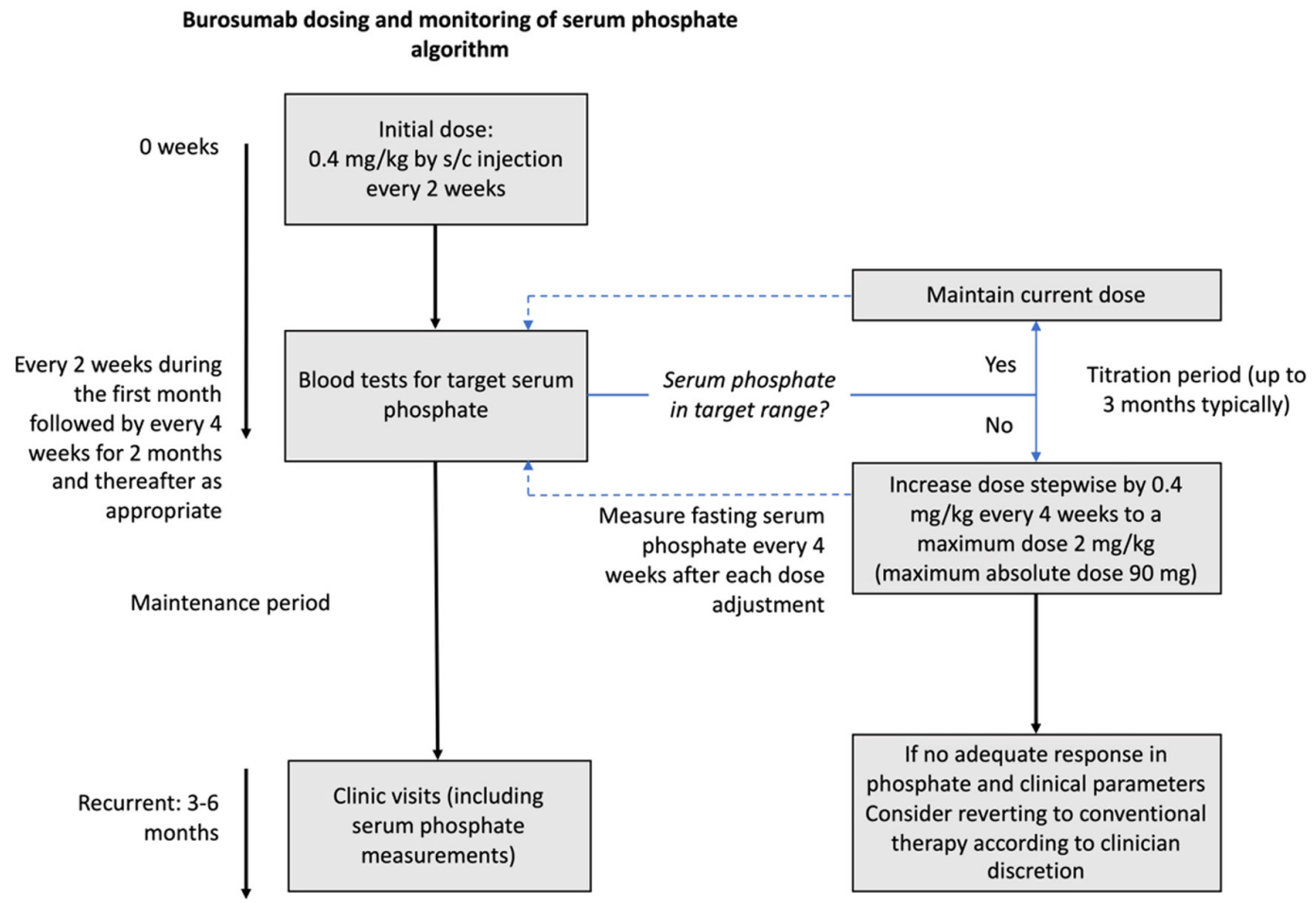

Increasing the
burosumab dose
should be considered
after two consequent
measurements of
phosphate levels
being below the lower
end of the normal
reference range (4
weeks). The increase
of the dose will also
depend on the
clinician's discretion
(checking patient's
weight).
Dose to be adjusted
according to the SPC._
if fasting serum
phosphate is above
the reference range
for age, the next dose
should be withheld
and the fasting serum
phosphate reassessed
within 4 weeks

Figure 1

Recommended burosumab dosing and titration regimen.

end of the normal reference range for age. Doses should be rounded to the nearest $10 \mathrm{mg}$ according to the Summary of Product Characteristics, with the option of rounding to $5 \mathrm{mg}$ (https://www.rcpch.ac.uk/about-us/specialtygroups-special-interest-groups\#british-paediatric-andadolescent-bone-group-bpabg) depending on clinician's decision, particularly in smaller infants and children.

Dose increase should be considered following two sequential measures of fasting serum phosphate below the normal range within 4 weeks, at the discretion of the clinician. Dose should be increased stepwise by $0.4 \mathrm{mg} / \mathrm{kg}$ up to a maximum of $90 \mathrm{mg}$ or $2 \mathrm{mg} / \mathrm{kg}$ (lowest value to be considered) and monitored via pre-dose fasting serum phosphate levels 4 weeks after the last dose adjustment. The dose should not be increased more frequently than every 4 weeks (https://www.ema.europa.eu/en/ documents/product-information/crysvita-epar-productinformation_en.pdf).

If fasting serum phosphate is above the reference range, the next dose should be withheld and the fasting serum phosphate reassessed within 4 weeks. The patient's fasting serum phosphate must be below the reference range for age in order to restart burosumab and, to avoid the risk of complications, this should be at the last dose at which fasting serum phosphate levels were at the lower limit of normal (https://www.ema.europa.eu/en/ documents/product-information/crysvita-epar-productinformation_en.pdf).

BPABG members agreed that in some cases, the recommended maximum dose of burosumab may not lead to an increase of serum phosphate close to the reference range within 3-6 months to produce clinical benefit. In these instances, the treatment should be reviewed, with the option of reverting to conventional therapy also being considered.

It is, however, important to note that in some circumstances fasting serum phosphate levels may increase substantially without reaching the normal range. In this situation, if there is clinical improvement, the patient could continue treatment with burosumab.

Once stable fasting serum phosphate levels are achieved during the titration period, follow-up may be reduced to every 3-6 months, depending on clinical need.

\section{Monitoring}

Fasting serum phosphate levels should be monitored every 3-6 months, along with clinical review by a bone disease specialist, broader auxology including head circumference (up to the age of 5 years), intermalleolar and intercondylar (c) 2020 The authors Published by Bioscientifica Ltd
This work is licensed under a Creative Commons Attribution-NonCommercial-NoDerivatives 4.0 elfternationad dicense.ifica . com at 04/26/2023 07:32:03AM 
distances. Radiology investigations should also be used to support clinical decision-making as appropriate.

A comprehensive set of blood and urine tests should be taken every 3-6 months but may be required more frequently depending on dose modifications. This includes bone profile, renal profile, PTH, 25(OH)D and spot urine calcium: creatinine ratio and TmP/GFR.

Renal ultrasound should be performed at baseline and 6 months after commencing treatment with burosumab and yearly thereafter to screen for and monitor development and progression of nephrocalcinosis. On an annual basis, blood pressure should be checked. A clinical assessment of skeletal deformity, including craniosynostosis when age appropriate, should also be made at every clinic visit and, if required, referral to relevant specialist surgical teams for assessment. Twice yearly dental assessment by a suitable professional is recommended in order to screen and manage dental infections and periodontitis.

Additional tests which should be considered include mobility assessment (e.g. via the 6-min walk test), pain scoring, quality-of-life assessments and baseline hearing tests in line with local policy. A summary of key monitoring parameters is shown in Table 1.

\section{Adverse effects}

In clinical trials with burosumab the most common adverse drug reactions (ADR) reported in paediatric patients up to 64 weeks were injection site reactions (57\%), headache $(54 \%)$, pain in extremity $(42 \%)$, a reduction in 25-hydroxyvitamin D concentration (28\%), rash (23\%), toothache (19\%), tooth abscess (14\%), myalgia (14\%), and dizziness (11\%) (Burosumab SmPC).

Adverse effects to treatment with burosumab should be subject to active collection and reporting. Disease symptoms should be monitored through clinical examination (weight, mobility, pain) and radiography, and any unexpected effects reported. Any sign of local reactions should be reported. For example, in the UK, healthcare professionals are asked to report any suspected adverse reactions via the national Yellow Card scheme (https://yellowcard.mhra.gov.uk/).

\section{Pregnancy}

The possibility of pregnancy should be considered before initiation of burosumab. The SmPC for burosumab states that it is not recommended during pregnancy and in women of childbearing potential not using contraception (https://www.ema.europa.eu/en/documents/productinformation/crysvita-epar-product-information_en.pdf). In any post-menarchal female patient with amenorrhoea, a pregnancy test should be discussed and undertaken.

\section{Expectations for multidisciplinary care arrangements}

It is expected that the patient will be initially assessed by a tertiary metabolic bone specialist in a hospital which is

Table 1 Monitoring frequency.

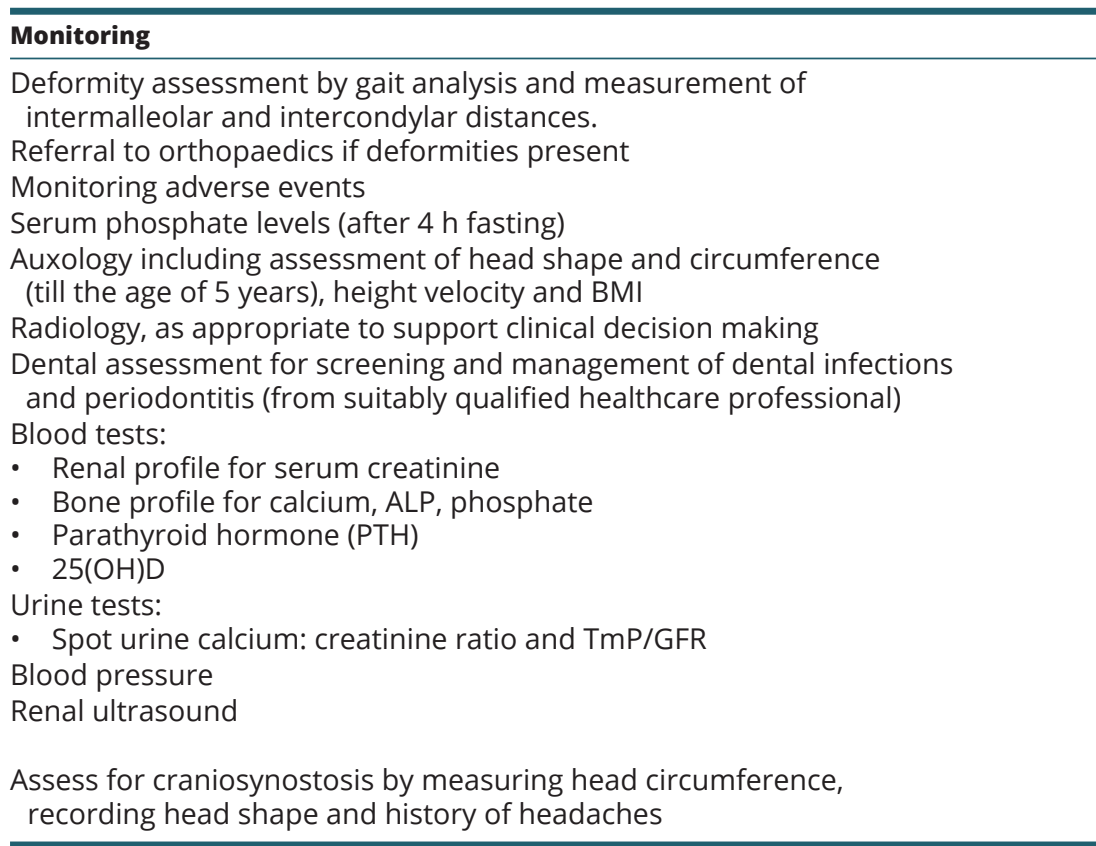

\section{Frequency}

At every clinical visit

Every 3-6 months 6 months at clinic visit

Every 6 months

Minimum every 6 months (more frequently at clinician's discretion or if serum phosphate levels are low)

Annually

At baseline and 6 months after commencing treatment and annually thereafter

At every clinical visit in those under 5 years https://ec.bioscientifica.com https://doi.org/10.1530/EC-20-0291 (c) 2020 The authors Published by Bioscientifica Ltd
This work is licensed under a Creative Commons Attribution-NonCommercial-NoDerivatives 4.0 elnternational License $;$ fica com at $04 / 26 / 2023 \quad 07: 32: 03 \mathrm{Am}$ 
a recognised centre of excellence for rare bone disorders. The decision to start, cease, or alter treatment (including dose escalation) must be made by the metabolic bone specialist and authorised hospital. In most circumstances the supply of burosumab will be via the home-care route where close liaison with the pharmacy department at the tertiary specialist hospital centre and the patient's local hospital will be required to ensure continuity of supply and appropriate reimbursement by healthcare providers. Administration of the injection may be in the local hospital, the tertiary hospital or at home by community/ outreach nurses. Monitoring can be undertaken locally but results should be shared with the initiating clinician. The patient should however be assessed by a metabolic bone specialist at least on an annual basis. Adverse events should be reported to both the initiating physician and the local clinician, and via the relevant national reporting system.

\section{Conclusions}

These recommendations from BPABG support the standardised introduction of the novel FGF23 inhibitor, burosumab, following NICE approval into clinical practice for treatment naïve paediatric and adolescent XLH patients and those who have previously been on conventional therapy. The availability of tertiary bone centres greatly facilitates the introduction of burosumab into the care pathway and also enables the health care provider to monitor the use and uptake of burosumab.

\section{Supplementary materials}

This is linked to the online version of the paper at https://doi.org/10.1530/ EC-20-0291.

\section{Declaration of interest}

The authors declare that there is no conflict of interest that could be perceived as prejudicing the impartiality of the research reported.

\section{Funding}

The authors disclosed receipt of financial support for the publication of this article by Kyowa Kirin International as well as funding for phase 2 and phase 3 clinical trial and fees for consultancy, scientific advisor and education and training.

\section{Acknowledgements}

The authors acknowledge support from MAP BioPharma for their editorial role in the development of this manuscript as well as the BPABG members who participated in the consultation process.

\section{References}

1 Haffner D, Emma F, Eastwood DM, Duplan MB, Bacchetta J, Schnabel D, Wicart P, Bockenhauer D, Santos F, Levtchenko E, et al. Clinical practice recommendations for the diagnosis and management of X-linked hypophosphataemia. Nature Reviews: Nephrology 201915 435-455. (https://doi.org/10.1038/s41581-0190152-5)

2 Imel EA \& Carpenter TO. A practical clinical approach to paediatric phosphate disorders. Endocrine Development 201528 134-161. (https://doi.org/10.1159/000381036)

3 Linglart A, Biosse-Duplan M, Briot K, Chaussain C, Esterle L, Guillaume-Czitrom S, Kamenicky P, Nevoux J, Prié D, Rothenbuhler A, et al. Therapeutic management of hypophosphatemic rickets from infancy to adulthood. Endocrine Connections 20143 R13-R30. (https://doi.org/10.1530/EC-130103)

4 Carpenter TO, Imel EA, Ruppe MD, Weber TJ, Klausner MA, Wooddell MM, Kawakami T, Ito T, Zhang X, Humphrey J, et al. Randomized trial of the anti-FGF23 antibody KRN23 in X-linked hypophosphatemia. Journal of Clinical Investigation 2014124 1587-1597. (https://doi.org/10.1172/JCI72829)

5 Imel EA, Glorieux FH, Whyte MP, Munns CF, Ward LM, Nilsson O, Simmons JH, Padidela R, Namba N, Cheong HI, et al. Burosumab versus conventional therapy in children with X-linked hypophosphataemia: a randomised, active-controlled, open-label, phase 3 trial. Lancet 2019393 2416-2427. (https://doi.org/10.1016/ S0140-6736(19)30654-3)

6 Whyte MP, Carpenter TO, Gottesman GS, Mao M, Skrinar A, San Martin J \& Imel EA. Efficacy and safety of burosumab in children aged 1-4 years with X-linked hypophosphataemia: a multicentre, open-label, phase 2 trial. Lancet: Diabetes and Endocrinology 20197 189-199. (https://doi.org/10.1016/S22138587(18)30338-3)

7 Carpenter TO, Imel EA, Holm IA, Jan de Beur SM \& Insogna KL. A clinician's guide to X-linked hypophosphatemia. Journal of Bone and Mineral Research 201126 1381-1388. (https://doi.org/10.1002/ jbmr.340)

8 Fukumoto S, Ozono K, Michigami T, Minagawa M, Okazaki R, Sugimoto T, Takeuchi Y \& Matsumoto T. Pathogenesis and diagnostic criteria for rickets and osteomalacia - proposal by an expert panel supported by the Ministry of Health, Labour and Welfare, Japan, the Japanese Society for Bone and Mineral Research, and the Japan Endocrine Society. Journal of Bone and Mineral Metabolism 201533 467-473. (https://doi.org/10.1007/s00774-015-0698-7)

Received in final form 29 September 2020

Accepted 8 October 2020

Accepted Manuscript published online 8 October 2020 https://ec.bioscientifica.com https://doi.org/10.1530/EC-20-0291 (c) 2020 The authors Published by Bioscientifica Ltd
This work is licensed under a Creative Commons Attribution-NonCommercial-NoDerivatives 4.0 elfternationab ficense.ifica . com at 04/26/2023 07:32:03AM 